Original Research Paper

\title{
Something about the Mechanical Moment of Inertia
}

\author{
${ }^{1}$ Florian Ion T. Petrescu, ${ }^{2}$ Antonio Apicella, ${ }^{2}$ Aversa Raffaella, \\ ${ }^{3}$ Relly Victoria Petrescu, ${ }^{4}$ John Kaiser Calautit and ${ }^{5}$ Aniello Riccio \\ ${ }^{I}$ Department of Theory of Mechanisms and Robots, Bucharest Polytechnic University, Bucharest, Romania \\ ${ }^{2}$ Advanced Material Lab, Department of Architecture and Industrial Design, Second University of Naples, Aversa (CE) Italy \\ ${ }^{3}$ Department of Transport, Traffic and Logistics, Bucharest Polytechnic University, Bucharest, Romania \\ ${ }^{4}$ Department of Mechanical Engineering, University of Sheffield, Sheffield, United Kingdom \\ ${ }^{5}$ Second University of Naples, Naples, Italy
}

\section{Article history}

Received: 10-10-2016

Revised: $28-10-2016$

Accepted: 03-11-2016

Corresponding Author: Florian Ion T. Petrescu Department of Theory of Mechanisms and Robots, Bucharest Polytechnic

University, Bucharest, Romania Email: scipub02@gmail.com

\begin{abstract}
In this study, the relations to determining mass moments of inertia (mechanical) for different mass and mechanical inertia corresponding to geometric shapes, objects and profiles are explored. The formulas for calculating the mass moments of inertia (mechanical) for various bodies (various geometrical forms), to certain major axis indicated (as the axis of calculation) are presented. The total mass M of the body is used to determine the mass moment of inertia (mechanical). In the first part of the paper, an original method for determining the mass moment of inertia (mechanical) of the flywheel is presented. Mass moment of inertia (the whole mechanism) reduced at the crank (reduced to the element leader) consists in a constant mass inertia moment and one variable, to which we may include an additional mass moment of inertia flywheel, which aims to reduce the degree of unevenness of the mechanism and the default machine. The more the mass moment of inertia of the flywheel is increased the more the unevenness decreased and dynamic functioning of the mechanism is improved. Engineering optimization of these values can be realized through new relationships presented on the second paragraph of the article. Determining of the mass moment of inertia of the flywheel with the new method proposed is also based on the total kinetic energy conservation.
\end{abstract}

Keywords: Mass Moment of Inertia, Compute Axis, Unevenness, Flywheel Moment of Inertia, Energy Conservation

\section{Introduction}

Determination of mechanical moments of inertia (mass) is becoming increasingly important in modern industrial design and mechanical systems. The exact calculation of mechanical inertial moments no longer easily found today (only in older textbooks mechanics or mechanisms).

In this paper the authors aim initial to reminder the relations to determining mass moments of inertia (mechanical) for different mass and mechanical inertia corresponding to geometric shapes, objects and profiles.

The second part of this paper provides an overview of the equations for determining the mechanical mass moments of inertia.

The first part of the paper presents, an original method for determining the degree of irregularity of a machine (car) and a minimum mass moment of inertia the flywheel.
This is extremely useful for rotor dynamics that have high speed (Heat engines with internal and external combustion, electric motors, turbines, jet).

\section{Determining the Mechanical Moments of Inertia}

The mechanical mass moments of inertia (Antonescu, 2000; Pelecudi, 1985; Petrescu, 2012), of kinematic elements represent the mass to the rotational movement of these elements. Such as the known mechanical mass of a kinematic element that characterizes the translational movement of the respectively cinematic element.

For the kinetic energy of a system to be complete it is essential that the dynamic calculations to be taken into account and the rotation kinetic energy. This is due to movements of rotation of each kinematic element belonging to the mechanism or to the assembly. 
Dynamics is the only one that can simulate theoretical the real movement of a machine. In general, in the works of mechanics or mechanisms, motors, dynamics, etc., (scientific articles, books) there is no an emphasis on the determination of mass moments of inertia (mechanical) considering themselves too popular. In this article, different mechanical inertia corresponding to different geometric shapes, objects and profiles are presented. The formulas for the calculation of the moments by mass or mechanical inertia for various bodies (various shapes) are detailed.

It is noted with $\mathrm{M}$, the total mass of the element to which must be determined the inertia mechanical moment. Calculation formulas are displayed in the respective Fig. 1-13 (Petrescu, 2012).

\section{Determining the Mass of Inertia of the Flywheel $\left(\mathbf{J}_{\mathbf{v}}\right)$}

The uniformity work of a machine is characterized by the degree of irregularity $(\delta)$, defined by equation (1) (Antonescu, 2000; Pelecudi, 1985; Petrescu, 2012):

$$
\delta=\frac{\omega_{\max }-\omega_{\min }}{\omega_{\text {med }}}
$$

Average angular velocity is expressed by the relation (2):

$$
\omega_{\text {med }}=\frac{\omega_{\max }+\omega_{\min }}{2}
$$

From Equations 1 and 2, the maximum and minimum angular velocities are calculated using Equation 3 (Pelecudi, 1985; Petrescu, 2012):

$$
\left\{\begin{array}{l}
\omega_{\max }=\omega_{\text {med }} \cdot\left(1+\frac{\delta}{2}\right) \\
\omega_{\min }=\omega_{\text {med }} \cdot\left(1+\frac{\delta}{2}\right)
\end{array}\right.
$$

Equations 3 are squared to obtain a relational system as shown in Equations 4 (Pelecudi, 1985; Petrescu, 2012):

$$
\left\{\begin{array}{l}
\omega_{\max }^{2}=\omega_{m e d}^{2} \cdot\left(1+\frac{\delta}{2}\right)^{2}=\omega_{m}^{2} \cdot\left(1+\frac{\delta^{2}}{4}+\delta\right) \\
\omega_{\min }^{2}=\omega_{\text {med }}^{2} \cdot\left(1+\frac{\delta}{2}\right)^{2} \omega_{m}^{2} \cdot\left(1+\frac{\delta^{2}}{4}-\delta\right)
\end{array}\right.
$$

The mass moment of inertia of the whole mechanism which is reduced to the crank (reduced to the leader element) $J^{*}$, usually consists of a constant mass inertia moment $J_{0}$ and one variable $J$, to which one may include an additional mass inertia moment, of a flywheel $J_{v}$, which aims to reduce the degree of unevenness of the mechanism (machine) (see relation 5). When $J_{v}$ is increased, the more the $\delta$ drops (Pelecudi, 1985). It is necessary to obtain an optimal value for $J_{v}$ :

$J^{*}=J_{0}+J_{v}+J$

From the total energy conservation, the entire mechanism (as long as it does not take into account elastic deformations and considering only the basic mechanics of rigid body, the potential energy it does not affect the operation and one uses just the kinematic energy) the relationship can be written as (6) (Petrescu, 2012):

$$
\left\{\begin{array}{l}
\frac{1}{2} \cdot J_{m}^{*} \cdot \omega_{m}^{2} \frac{1}{2} \cdot J_{\max }^{*} \cdot \omega_{\min }^{2}= \\
=\frac{1}{2} \cdot J_{\min }^{*} \cdot \omega_{\max }^{2}=\frac{1}{2} \cdot J^{*} \cdot \omega^{2} \\
J_{m}^{*} \cdot \omega_{m}^{2}=J_{\max }^{*} \cdot \omega_{\min }^{2}=J_{\min }^{*} \cdot \omega_{\max }^{2}=J^{*} \cdot \omega^{2}
\end{array}\right.
$$

From Equation 6, only relation (7) is retained and is developed according to expression (5) (Petrescu, 2012). The equation is then written in the form of (8):

$$
\begin{aligned}
& J_{\max }^{*} \cdot \omega_{\min }^{2}=J_{\min }^{*} \cdot \omega_{\max }^{2} \\
& \left(J_{0}+J_{v}+J_{\max }\right) \cdot \omega_{\min }^{2}=\left(J_{0}+J_{v}+J_{\min }\right) \cdot \omega_{\max }^{2}
\end{aligned}
$$

where, $\left(J_{\max }\right.$ and $\left.J_{\min }\right)$, are the maximum and minimum of $J$, from the expression (5). It explains $J_{v}$ from relation (8) and one obtains the expression (9) (Petrescu, 2012):

$$
J_{v}=\frac{J_{0} \cdot\left(\omega_{\min }^{2}-\omega_{\max }^{2}\right)+J_{\max } \cdot \omega_{\min }^{2}-J_{\min } \cdot \omega_{\max }^{2}}{\left(\omega_{\max }^{2}-\omega_{\min }^{2}\right)}
$$

Using the relations from the system (4) the expression (9) takes the form (10) (Petrescu, 2012):

$$
J_{v}=-J_{0}+\frac{J_{\max } \cdot\left(1-\frac{\delta}{2}\right)^{2}-J_{\min } \cdot\left(1+\frac{\delta}{2}\right)^{2}}{\left(1+\frac{\delta}{2}\right)^{2}-\left(1-\frac{\delta}{2}\right)^{2}}
$$

Relation (10) is reduced to the form (11) by processing the denominator and to the form (12), if processing and the fraction numerator (Petrescu, 2012):

$J_{v}=-J_{0}+\frac{J_{\max } \cdot\left(1-\frac{\delta}{2}\right)^{2}-J_{\min } \cdot\left(1+\frac{\delta}{2}\right)^{2}}{2 \cdot \delta}$ 


$$
J_{v}=-J_{0}-J_{m}+\frac{J_{\max }-J_{\min }}{2} \cdot\left(\frac{1}{\delta}+\frac{\delta}{4}\right)
$$

where, $J_{m}=\left(J_{\max }+J_{\min }\right) / 2$ and the maximum and minimum are found by would come close to zero of the derivation of $J$ (from expression 5) in relation of variable $\varphi$.

Knowing the maximum allowable $\delta$ one calculates with the relation (12) the minimum required (optimal value) of mass moment of inertia of the flywheel, $J_{v}$.

\section{Results and Discussion}

In this paper the authors aim initial to reminder the relations to determining mass moments of inertia (mechanical) for different mass and mechanical inertia corresponding to geometric shapes, objects and profiles.

The second part of this paper provides an overview of the equations for determining the mechanical mass moments of inertia. In the first part of the paper, an original method for determining the degree of irregularity of a machine (car) and a minimum mass moment of inertia to the flywheel was presented.

For the kinetic energy of a system to be complete it is essential that the dynamic calculations to be taken into account and the rotation kinetic energy.

This is due to movements of rotation of each kinematic element belonging to the mechanism or to the assembly. Dynamics is the only one that can simulate theoretical the real movement of a machine. In general, in the works of mechanics or mechanisms, motors, dynamics, etc., (scientific articles, books) there is no an emphasis on the determination of mass moments of inertia (mechanical) considering themselves too popular.

In this article, different mechanical inertia corresponding to different geometric shapes, objects and profiles are presented. The formulas for the calculation of the moments by mass or mechanical inertia for various bodies (various shapes) are detailed.

It is noted with $\mathrm{M}$, the total mass of the element to which must be determined the inertia mechanical moment. Calculation formulas are displayed in the respective Fig. 1-13 (Petrescu, 2012).

Going uniform of a machine (car) is characterized by unevenness, which should have a minimum value. In the second part of this paper, an overview of the relationships which is used to determine the mass moments of inertia (mechanical) is presented.

In the first part of the paper an original method for determining the degree of irregularity of a machine (car) and a minimum mass moment of inertia to the flywheel was discussed.

The advantage of the presented (proposed) method is the simplicity of its exposure and also the method on how to determine the value compared to other known methods (Antonescu, 2000; Pelecudi, 1985). The purpose of the work is to simplify the well-known methods (Wittenbauer, 1923) through a new tool and not to replace it.

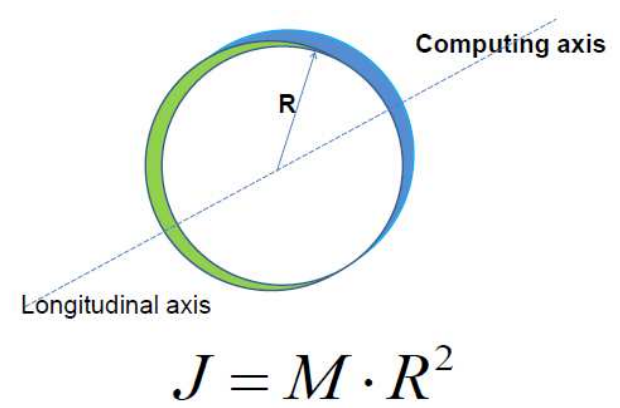

Fig. 1. Mass moment of inertia of a ring fixed (calculated) around the longitudinal axis of the ring

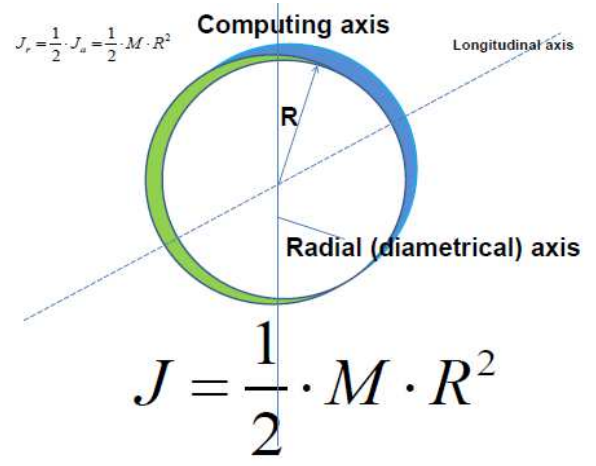

Fig. 2. Mass moment of inertia of a ring fixed around a radial (diametrical) axis of the ring

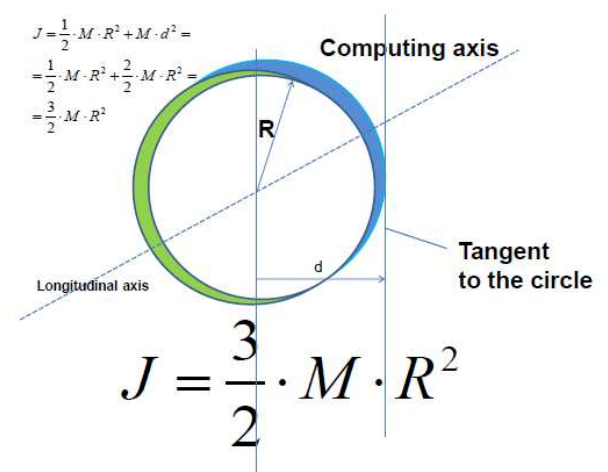

Fig. 3. Mass moment of inertia of a ring fixed around an axis tangent to the circle of the ring

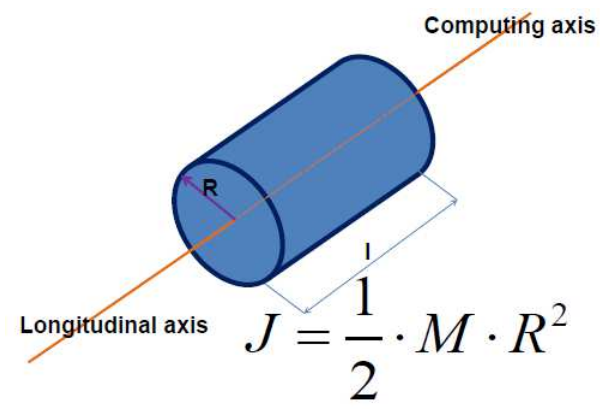

Fig. 4. Mass moment of inertia of a cylinder or a disc, fixed to the longitudinal axis of the cylinder or disc 


$$
\begin{aligned}
& J_{r}=\frac{1}{2} \cdot J_{a}=\frac{1}{2} \cdot \frac{1}{2} \cdot M \cdot R^{2}= \\
& =\frac{1}{4} \cdot M \cdot R^{2}
\end{aligned}
$$

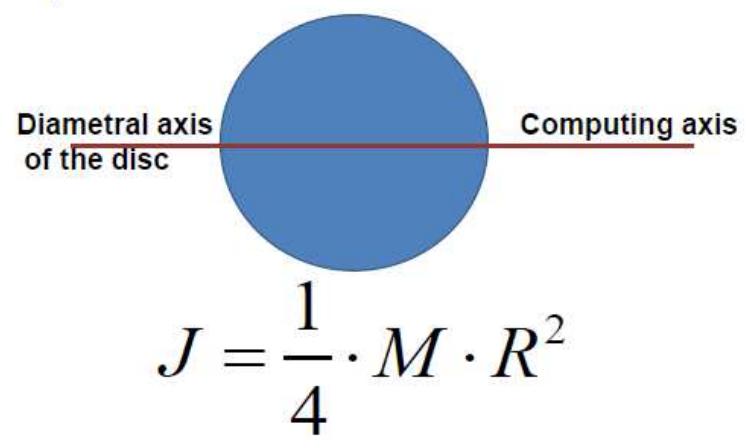

Fig. 5. Mass moment of inertia to a disc, determined to the radial (diametral) axis of the disc

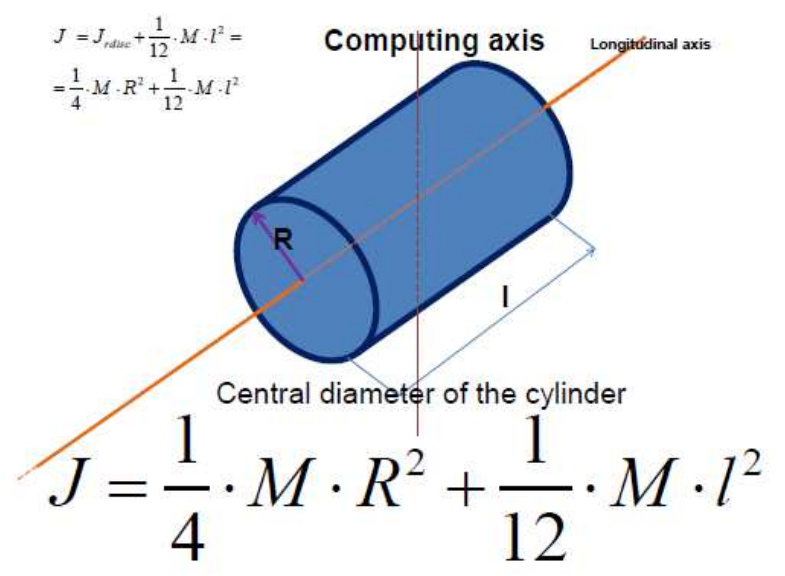

Fig. 6. Mass moment of inertia of a cylinder, determined in about an axis central diametral

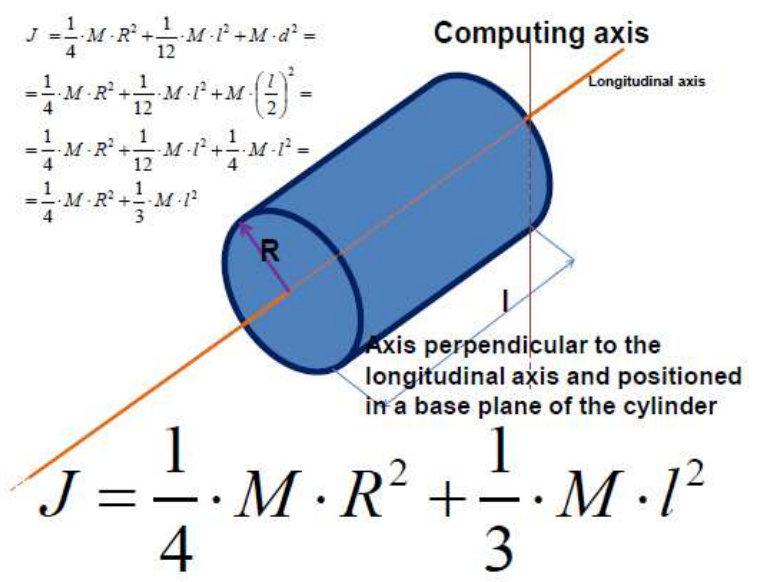

Fig. 7. Mass moment of inertia of the cylinder, caused about an axis lying in the plane of the end of the cylinder perpendicular to the longitudinal axis

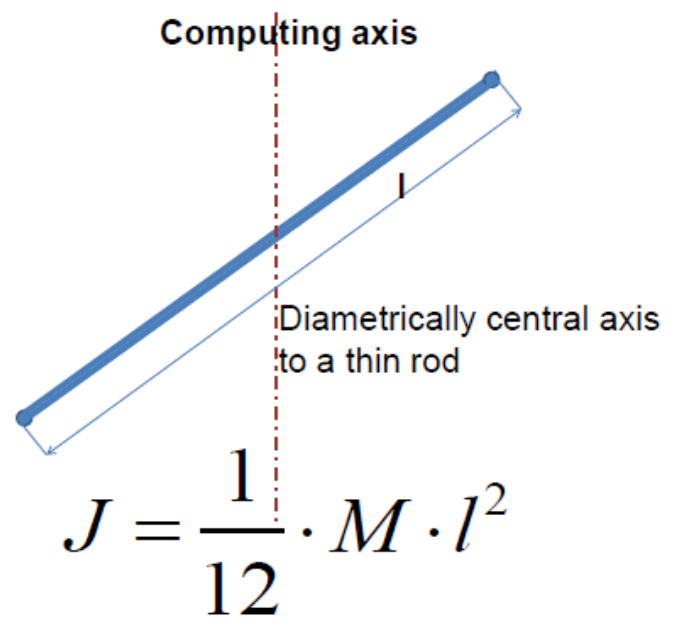

Fig. 8. Mass moment of inertia to a thin rod, led around an axis passing through a central diameter of the rod

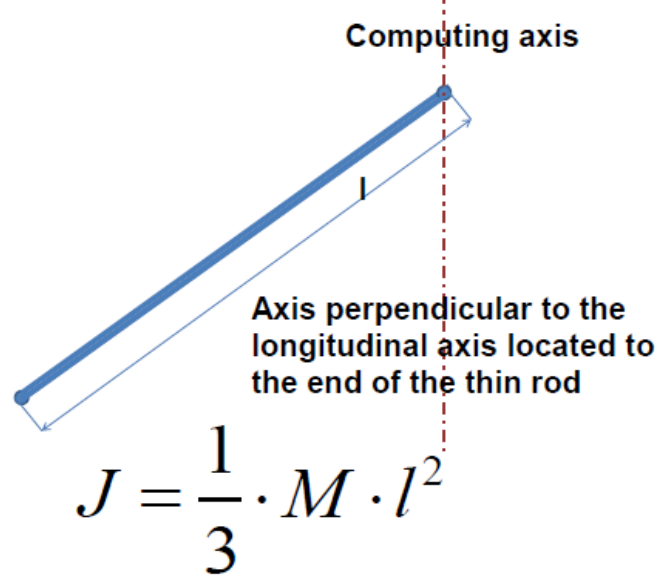

Fig. 9. Mass moment of inertia of a thin rod, determined about an axis located at one of the rod ends, perpendicular to the longitudinal axis of the stem

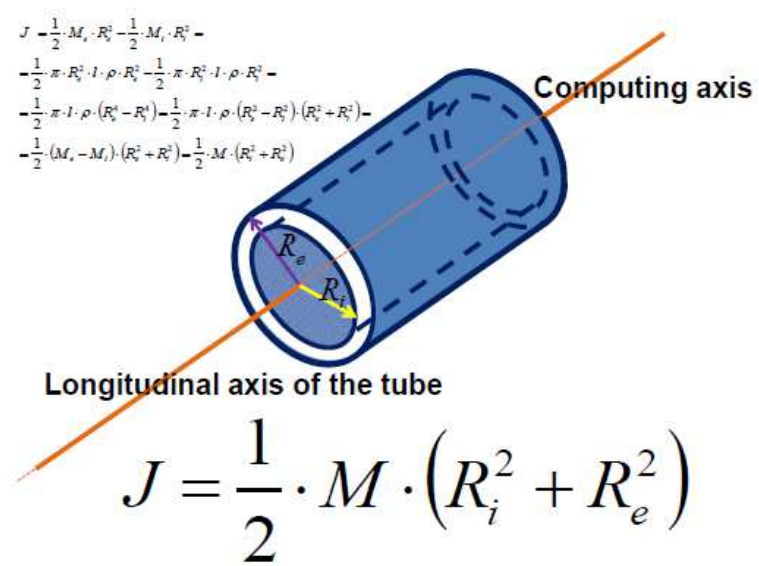

Fig. 10. Mass moment of inertia to a tube (pipe or annulus) determined about the longitudinal axis 


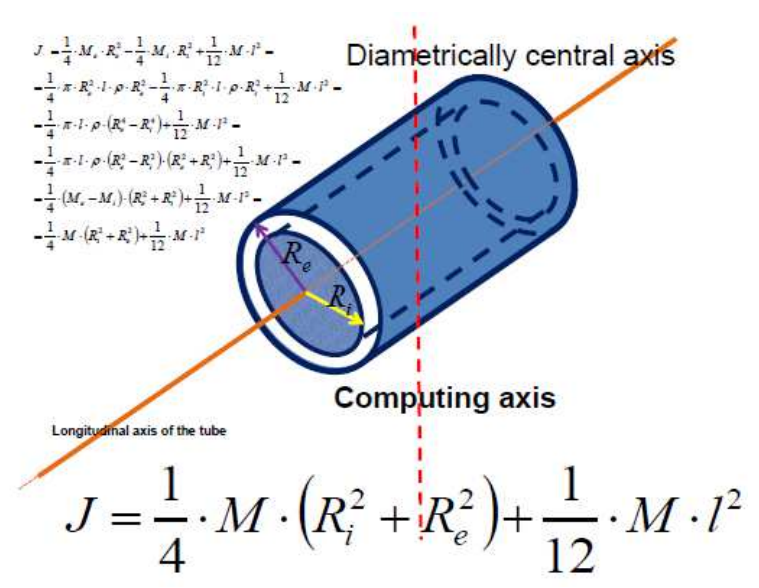

Fig. 11. Mass moment of inertia to a tube (pipe or annulus) determined about the diametrically central axis

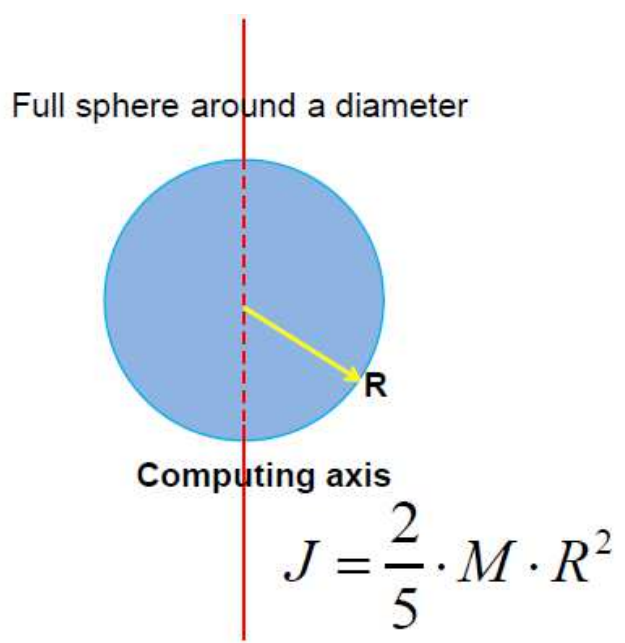

Fig. 12. Mass moment of inertia to a full sphere, determined around a diameter

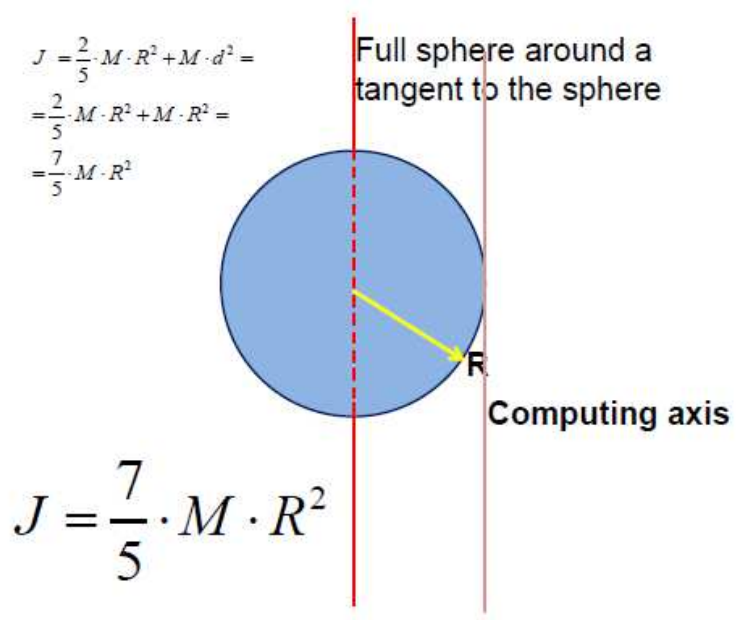

Fig. 13. Mass moment of inertia to a full sphere, determined around a tangent to the sphere

\section{Importance}

In this paper an original method for determining the degree of irregularity of a machine (car) and a minimum mass moment of inertia to the flywheel are presented. This is extremely useful for rotor dynamics that have high speed (Heat engines with internal and external combustion, electric motors, turbines, jet).

The authors aim initial to reminder the relations to determining mass moments of inertia (mechanical) for different mass and mechanical inertia corresponding to geometric shapes, objects and profiles.

The final part of this paper provides an overview of the equations for determining the mechanical mass moments of inertia, which have a lot of practical applications.

Determination of mass moments of inertia is required in any dynamic calculation of a machines, engine or lucrative (motor, car, vehicle, robot, machine tool, etc), (Petrescu, 2012; 2015; Petrescu and Petrescu, 2016).

Most spectacular is the use of formula from (Fig. 12) to determine the nano size of the elementary particles in moving dynamic (Petrescu and Calautit, 2016a).

Similarly was deduced even a third equation of the light, besides the two already known made by Einstein (Petrescu and Calautit, 2016b) and thus finding the photon sizes (mass and diameter) depending on the frequency of light.

\section{Conclusion}

The second part of this paper provides an overview of the equations for determining the mechanical mass moments of inertia.

In the first part is presented an original method for determining the degree of irregularity of a machine (car) and a minimum mass moment of inertia to the flywheel. This is extremely useful for rotor dynamics that have high speed.

Determination of mass moments of inertia is required in any dynamic calculation of a machines, engine or lucrative (motor, car, vehicle, robot, machine tool, etc), (Petrescu, 2012; 2015; Petrescu and Petrescu, 2016).

Most spectacular is the use of formula from (Fig. 12) to determine the nano size of the elementary particles in moving dynamic (Petrescu and Calautit, 2016a).

Similarly was deduced even a third equation of the light, besides the two already known made by Einstein (Petrescu and Calautit, 2016b) and thus finding the photon sizes (mass and diameter) depending on the frequency of light.

\section{Acknowledgement}

This text was acknowledged and appreciated by Dr. Veturia CHIROIU Honorific member of Technical Sciences Academy of Romania (ASTR) PhD supervisor in Mechanical Engineering and by the collectives of mechanisms of TMR departments from Bucharest, Brasov, Cluj-Napoca, Craiova, Iasi, from Romania, whom we thank and in this way. 


\section{Funding Information}

Research contract: Contract number 36-5-4D/1986 from 24IV1985, beneficiary CNST RO (Romanian National Center for Science and Technology) Improving dynamic mechanisms.

Research contract: Contract number 27-7-7/1987, beneficiary ICCM ROU (Romania Central Institute of Machine Construction), Research to reduce curb weight and increase reliability of resistance structures for buses.

\section{Author's Contributions}

All the authors contributed equally to prepare, develop and carry out this work.

\section{Ethics}

This article is original. Author declares that are not ethical issues that may arise after the publication of this manuscript.

\section{References}

Antonescu, P., 2000. Mechanisms and Manipulators. 1st Edn., Printech Publisher, Bucharest, Romanian.
Pelecudi, C., 1985. Mechanisms. 1st Edn., Didactic and Pedagogical Publisher, Bucharest, Romanian version.

Petrescu, F.I. and J.K. Calautit, 2016a. About nano fusion and dynamic fusion. Am. J. Applied Sci., 13: 261-266. DOI: 10.3844/ajassp.2016.261.266

Petrescu, F.I. and J.K. Calautit, 2016b. About the light dimensions. Am. J. Applied Sci., 13: 321-325. DOI: 10.3844/ajassp.2016.321.325

Petrescu, F.I. and R.V. Petrescu, 2016. Dynamic cinematic to a structure 2R. Rev. Geintec-Gestao Inovacao E Tecnol., 6: 3143-3154. DOI: $10.7198 /$ geintec.v6i2.371

Petrescu, F.I., 2012. Theory of Mechanisms. 1st Edn., Create Space publisher, USA, ISBN-10: 9781479293629, pp: 284.

Petrescu, F.I., 2015. Machine motion equations at the internal combustion heat engines. Am. J. Eng. Applied Sci., 8: 127-137. DOI: 10.3844/ajeassp.2015.127.137

Wittenbauer, F., 1923. Graphische Dynamik: Ein Lehrbuch für Studierende und Ingenieure mit zahlreichen Anwendungen und Aufgaben, 1st Edn., Julius Springer, Berlin, pp: 797. 